\title{
Randomized Trial Comparing Proactive, High-Dose versus Reactive, Low-Dose Intravenous Iron Supplementation in Hemodialysis (PIVOTAL): Study Design and Baseline Data
}

\author{
Iain C. Macdougalla Claire White ${ }^{a}$ Stefan D. Anker ${ }^{b} \quad$ Sunil Bhandari ${ }^{c}$ \\ Kenneth Farrington ${ }^{d, e}$ Philip A. Kalra ${ }^{f}$ John J.V. McMurrayg Heather Murray ${ }^{\text {h }}$ \\ Retha Steenkamp ${ }^{i}$ Charles R.V.Tomsonj David C. Wheeler ${ }^{k}$ \\ Christopher G. Winearls' lan Ford $^{\text {h }}$ on behalf of the PIVOTAL Trial investigators

\begin{abstract}
a Department of Renal Medicine, King's College Hospital, Denmark Hill, London, UK; ' Division of Cardiology and Metabolism, Department of Cardiology (CVK), and Berlin-Brandenburg Center for Regenerative Therapies (BCRT), German Centre for Cardiovascular Research (DZHK) Partner Site Berlin, Charité Universitätsmedizin Berlin, Berlin, Germany; ${ }^{C}$ Hull and East Yorkshire Hospitals and Hull York Medical School, Hull, UK; ${ }^{d}$ Lister Hospital, Stevenage,

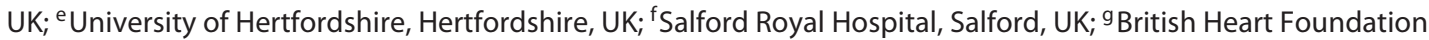
Cardiovascular Research Centre, University of Glasgow, Glasgow, UK; ${ }^{\text {h }}$ Robertson Centre for Biostatistics, University of Glasgow, Glasgow, UK; 'UK Renal Registry, Southmead Hospital, Bristol, UK; ' ${ }^{j}$ Freeman Hospital, Newcastle upon Tyne, UK; ' 'University College London, London, UK; 'Oxford Kidney Unit, The Churchill, Oxford University Hospitals NHS
\end{abstract} \\ Foundation Trust, Oxford, UK
}

\section{Keywords}

Anemia $\cdot$ Chronic kidney disease $\cdot$ Hemodialysis ·

Intravenous iron · Iron sucrose

\begin{abstract}
Background: Intravenous (IV) iron supplementation is a standard maintenance treatment for hemodialysis (HD) patients, but the optimum dosing regimen is unknown. Methods: PIVOTAL (Proactive IV irOn Therapy in hemodiALysis patients) is a multicenter, open-label, blinded endpoint, randomized controlled (PROBE) trial. Incident HD adults with a serum ferritin $<400 \mu \mathrm{g} / \mathrm{L}$ and transferrin saturation (TSAT) levels $<30 \%$ receiving erythropoiesis-stimulating agents (ESA) were eligible. Enrolled patients were randomized to a proactive, high-dose IV iron arm (iron sucrose $400 \mathrm{mg} / \mathrm{month}$
\end{abstract}

\begin{tabular}{ll}
\hline KARGER & ( 2018 The Author(s) Karger \\
& Published by S. Karger AG, Basel Open access \\
E-Mail karger@karger.com & This article is licensed under the Creative Commons Attribution- \\
www.karger.com/ajn & $\begin{array}{l}\text { NonCommercial-NoDerivatives 4.0 International License (CC BY- } \\
\text { NC-ND) (http://www.karger.com/Services/OpenAccessLicense). } \\
\text { Usage and distribution for commercial purposes as well as any dis- } \\
\text { tribution of modified material requires written permission. }\end{array}$
\end{tabular}

unless ferritin $>700 \mu \mathrm{g} / \mathrm{L}$ and/or TSAT $\geq 40 \%$ ) or a reactive, low-dose IV iron arm (iron sucrose administered if ferritin $<200 \mu \mathrm{g} / \mathrm{L}$ or TSAT $<20 \%)$. We hypothesized that proactive, high-dose IV iron would be noninferior to reactive, low-dose IV iron for the primary outcome of first occurrence of nonfatal myocardial infarction (MI), nonfatal stroke, hospitalization for heart failure or death from any cause. If noninferiority is confirmed with a noninferiority limit of 1.25 for the hazard ratio of the proactive strategy relative to the reactive strategy, a test for superiority will be carried out. Secondary outcomes include infection-related endpoints, ESA dose requirements, and quality-of-life measures. As an event-driven trial, the study will continue until at least 631 primary outcome events have accrued, but the expected duration of follow-up is 2-4 years. Results: Of the 2,589 patients screened across 50 UK sites, 2,141 (83\%) were randomized. At base-
Prof. Ian Ford

Robertson Centre for Biostatistics

University of Glasgow, Level 11, Boyd Orr Building

Glasgow G12 8QQ (UK)

E-Mail Ian.Ford@glasgow.ac.uk 
line, $65.3 \%$ were male, the median age was 65 years, and $79 \%$ were white. According to eligibility criteria, all patients were on ESA at screening. Prior stroke and $\mathrm{MI}$ were present in 8 and $9 \%$ of the cohort, respectively, and $44 \%$ of patients had diabetes at baseline. Baseline data for the randomized cohort were generally concordant with recent data from the UK Renal Registry. Conclusions: PIVOTAL will provide important information about the optimum dosing of IV iron in HD patients representative of usual clinical practice. Trial Registration: EudraCT number: 2013-002267-25.

(C) 2018 The Author(s)

Published by S. Karger AG, Basel

\section{Introduction}

Since the introduction of erythropoiesis-stimulating agents (ESAs) for the management of anemia in chronic kidney disease, intravenous (IV) iron has been widely used, particularly in hemodialysis (HD) patients where the average daily losses of iron typically exceed the oral absorption of iron $[1,2]$. However, the maintenance IV iron regimen varies widely from one country to another, and indeed among dialysis centers in the same country. For example, much larger quantities of IV iron are administered to HD patients in the United States, while IV iron is used very sparingly in Japanese HD patients [3]. Practice in the remainder of the world, including Europe, Australia, and Canada, is somewhere in the middle.

The reason for this variation is the lack of any evidence-base to inform clinical practice, as well as the fact that there are both advantages and disadvantages to the use of IV iron. The advantages of using a higher dosing regimen of IV iron include lower ESA dosage requirements. A number of landmark trials have demonstrated that higher ESA doses, targeting higher hemoglobin levels, are associated with adverse clinical outcomes [411]. In 1996, the Normal Hematocrit Cardiac Trial was halted prematurely because of concerns in the arm randomized to normalization of hematocrit (i.e., 42\%); subsequent analysis confirmed that the higher hematocrit was associated with an approximately $30 \%$ increase in the risk of death or nonfatal myocardial infarction (MI) [10]. Data from the Correction of Hemoglobin and Outcomes in Renal Insufficiency trial supported an association between higher ESA doses and the primary composite endpoint of death, heart failure (HF) hospitalization, stroke, or MI [5]. A further post hoc analysis of the Correction of Hemoglobin and Outcomes In Renal Insufficiency data demonstrated that the cardiovascular toxicity of higher epoetin-alfa doses was separable from hemoglobin concentrations [6]. Finally, a two-fold increase in stroke was independently associated with the use of darbepoetin alfa in the randomized placebo-controlled Trial to Reduce Cardiovascular Events with Aranesp Therapy $[8,9]$. In addition to cost savings, reduced ESA use afforded by higher doses of iron may reduce the risk of cardiovascular events observed in the aforementioned trials. Independent of ESA use, dialysis patients are at high risk for cardiovascular events and there is also strong evidence in patients with HF documenting improvements in symptoms and in left ventricular systolic function with IV iron repletion [12-16].

Conversely, there are concerns about the potential safety of high doses of IV iron. These have been reviewed extensively and were the focus of a Kidney Disease: Improving Global Outcomes Controversies Conference on Iron Management [1]. These concerns include the potential for increased oxidative stress due to hydroxyl radical generation observed in some studies, which could exacerbate cardiovascular toxicity [1, 17-19]. Similarly, there is laboratory evidence that IV iron can enhance bacterial proliferation and reduce neutrophil killing of bacteria, generating concerns about an increased risk of infection $[1,18,20]$. Observational data on cardiovascular outcomes and infection-related events associated with highdose IV iron use are conflicting [21, 22]. In any case, despite adjusting for confounders, observational studies are subject to both known and unknown residual confounding. A recent meta-analysis of randomized controlled trials comparing high-dose versus low-dose IV iron in patients undergoing HD generated only 7 studies worthy of inclusion, and all of these had limitations, with regard to sample size and duration of follow-up [21,23].

Thus, there has been a need for a scientifically rigorous, adequately powered, randomized controlled trial with sufficient duration of follow-up. To fulfill this need, the Proactive IV irOn Therapy in hemodiALysis patients (PIVOTAL) trial was conceived. This study was designed to compare the effects of a proactive, high-dose IV iron regimen and reactive, low-dose IV iron regimen among incident ( $<12$ months) HD patients. We hypothesized that proactive, high-dose IV iron would be noninferior to reactive, low-dose IV iron for the primary outcome of first occurrence of nonfatal MI, nonfatal stroke, hospitalization for HF, or death from any cause. PIVOTAL was also designed to provide insights on how these iron therapy approaches affect ESA dose requirements, red blood cell) transfusions, complications of HD treatment, quality of life, and relevant laboratory 
Table 1. PIVOTAL trial endpoints

Primary

A composite of nonfatal MI, nonfatal stroke, hospitalization for HF or death from any cause, analyzed as time-to-first event

\begin{tabular}{ll}
\hline Secondary (efficacy) & Secondary (safety) \\
\hline - Total MI, stroke, hospitalization for HF and deaths, including first and & - Vascular access thrombosis \\
recurrent events & - All-cause hospitalization \\
- All-cause death & - Hospitalization for infection \\
- First composite cardiovascular event (MI, stroke, and hospitalization for HF) & - Time to first and number of infection episodes \\
- Fatal or nonfatal MI & \\
- Fatal or nonfatal stroke & \\
- Heart failure hospitalization & \\
- ESA dose requirements & \\
- Transfusion requirements & \\
- EuroQol-5D (EQ-5D) & \\
- Kidney Disease Quality of Life (KDQOL) Instrument &
\end{tabular}

Tertiary

- Cumulative dose of iron

- Hemoglobin level

- Platelet count

- Serum ferritin level

- Serum albumin level

- TSAT

HF, heart failure; MI, myocardial infarction; TSAT, transferrin saturation.

Fig. 1. PIVOTAL trial design. ESA, erythropoiesis-stimulating agents; $\mathrm{HD}$, hemodialysis; HF, heart failure; IV, intravenous; MI, myocardial infarction; TSAT, transferrin saturation.

\begin{tabular}{|c|c|c|}
\hline \multirow{3}{*}{$\begin{array}{c}\text { New to HD } \\
\text { On } 0-12 \text { months })\end{array}$} & Proactive, high-dose IV iron arm & \multirow{4}{*}{$\begin{array}{c}\text { - } \\
\text { I } \\
\text { endpoint events } \\
\text { I } \\
\text { I.e., all-cause } \\
\text { mortality, } \mathrm{Ml} \text {, } \\
\text { stroke, or HF } \\
\text { I hospitalization) } \\
\text { I }\end{array}$} \\
\hline & $\begin{array}{l}\text { IV iron } 400 \mathrm{mg} / \mathrm{month} \text { (withhold if ferritin }>700 \mu \mathrm{g} / \mathrm{L} ; \\
\text { TSAT >40\%) }\end{array}$ & \\
\hline & IV iron only administered if ferritin $<200 \mu \mathrm{g} / \mathrm{L}$ or TSAT $<20 \%$ & \\
\hline $\begin{array}{l}\leq 4 \text { weeks } \\
\text { screening }\end{array}$ & Follow-up period with monthly visits ( $\sim 2-4$ years per patient) & \\
\hline
\end{tabular}

biomarkers. This paper reports baseline data on all randomized subjects and sets out the protocol for management of subjects following randomization and throughout follow-up.

\section{Material and Methods}

\section{Study Design}

PIVOTAL is a multicenter prospective, open-label, blinded endpoint, 2-arm randomized controlled trial among incident HD patients undergoing HD for $<12$ months (EudraCT number 2013002267-25). Following a screening period of up to 4 weeks, patients were randomized to receive a proactive, high-dose IV iron or a reactive, low-dose IV iron regimen (Fig. 1).

The primary efficacy measure of the PIVOTAL trial is the composite of nonfatal MI, nonfatal stroke, hospitalization for HF, or death from any cause, analyzed as time to first event. All suspected, relevant, nonfatal events and deaths are adjudicated by a blinded Endpoint Adjudication Committee. Secondary endpoints include the individual components of the primary endpoint, ESA dose requirements, and infection-related measures. Table 1 details the study endpoints. Patients will attend monthly follow-up visits until the required number of primary endpoint events has accrued (see power calculations). It was expected that the trial would take approximately 4 years to complete, allowing approximately 2 years for recruitment and approximately $2-4$ years follow-up for each patient. 
Table 2. Inclusion and exclusion criteria

\begin{tabular}{ll}
\hline Inclusion criteria & Exclusion criteria \\
\hline - Age $>18$ years & - Life expectancy $<12$ months per the judgement of the investigator \\
- Patients established on a chronic HD program for end-stage & - Living-donor transplant scheduled within 12 months \\
renal failure & - Scheduled to switch to peritoneal dialysis or home HD \\
- Clinically stable per the judgment of the investigator & - CRP $>50$ mg/L \\
- 0 - 12 months since commencing HD & - Active infection \\
- Patients who have switched to HD from peritoneal dialysis or & - Current active malignancy (i.e., progressive untreated cancer or \\
have received previous HD or renal transplants are eligible to & current treatment with cytotoxic chemotherapy) \\
enter the study & - Known HIV, active hepatitis B (i.e., HBV DNA positive), or active \\
- Ferritin $<400 \mu g / L$ & hepatitis C (i.e., HCV RNA is positive) \\
- TSAT $<30 \%$ & - Chronic liver disease and/or screening ALT or AST $>3 \times$ ULN \\
- On ESA therapy & - Advanced HF (i.e., NYHA class IV) \\
- Written informed consent & - Pregnancy or breast feeding \\
& - History of acquired iron overload \\
& - Previous severe hypersensitivity reactions to IV iron sucrose \\
& - Compromised ability to give written informed consent and/or to \\
& comply with study procedures \\
\hline
\end{tabular}

a Patients with basal cell or squamous cell carcinoma of the skin or cervical intraepithelial neoplasia and those on "prophylactic" maintenance treatment for cancer were permitted to enroll.

ALT, alanine aminotransferase; AST, aspartate aminotransferase; HBV, hepatitis B virus; HCV, hepatitis C virus; HIV, human immunodeficiency virus; NYHA, New York Heart Association; RNA, ribonucleic acid; ULN, upper limit of normal.

\section{Eligibility}

The eligibility criteria for the trial were designed to encourage enrollment of a real-world cohort. Adults with end stage renal disease started on chronic HD but treated for less than 12 months were eligible if they were on ESA therapy and had a serum ferritin level $<400 \mu \mathrm{g} / \mathrm{L}$ and a transferrin saturation (TSAT) $<30 \%$. Patients who were consented within 12 months of commencing chronic HD but failed screening only because the ferritin levels were too high could be randomized only after their ferritin level had dropped to $<400 \mu \mathrm{g} / \mathrm{L}$, provided they did not exceed 18 months of HD. Routine IV iron therapy was stopped at the time of consent. Patients were excluded if they had evidence of an active infection or other conditions in which iron therapy might pose safety concerns. Table 2 details the full eligibility criteria for the trial.

\section{Screening}

Written informed consent was obtained from potentially eligible patients, and routine therapy with IV iron was discontinued. At the screening visit, eligibility was assessed by the collection of demographic information, review of the medical history, and laboratory measurements. Patients meeting eligibility criteria at the initial screening visit were transitioned from their routine iron regimen to the study regimen without any specified period off iron therapy. Patients who failed initial screening, particularly those not meeting ferritin or TSAT inclusion criteria, could be re-screened and randomized after meeting eligibility criteria.

\section{Randomization and Intervention}

Patients were randomized within 4 weeks of passing the screening requirements and were assigned to one of 2 treatment arms in a 1:1 ratio using a web-based randomization system. Randomization was based on randomly generated permuted blocks of variable sizes allocated within study sites and was stratified by the type of vascular access (dialysis catheter versus A-V fistulae) in use at the time of randomization, diabetic status, and time on dialysis at the time of initial screening (0-4 vs. 5-12 months).

In both treatment arms, monthly iron doses were determined following monthly assessments of serum ferritin and TSAT. Iron (if not withheld) was administered during the first HD sessions in the week following the monthly assessment of the iron indices. All iron was administered as undiluted iron sucrose as a slow bolus injection or by IV infusion according to standard practice of the individual center. Patients assigned to the proactive, high-dose IV iron arm were to receive IV iron sucrose $200 \mathrm{mg}$ during each of the 3 dialysis sessions at the start of the study, and during each of the first 2 dialysis sessions of the week following the monthly blood tests for all subsequent months (i.e., $400 \mathrm{mg}$ per month). If monthly testing demonstrated ferritin $>700 \mu \mathrm{g} / \mathrm{L}$ and/or TSAT $\geq 40 \%$, IV iron was withheld for the month.

Patients randomized to the reactive, low-dose IV iron arm received iron based on a pre-specified dosing schema, which permitted the administration of iron only if patients were deemed "iron deficient" as assessed by serum ferritin levels and TSAT. If monthly testing demonstrated (1) ferritin $>200 \mu \mathrm{g} / \mathrm{L}$ and TSAT $>20 \%$, (2) ferritin $>700 \mu \mathrm{g} / \mathrm{L}$, or (3) TSAT $\geq 40 \%$, iron was not administered that month. Provided that TSAT was $<40 \%$, patients with ferritin $<100 \mu \mathrm{g} / \mathrm{L}$ received iron sucrose $200 \mathrm{mg}$ during the first 2 dialysis sessions of the week; if ferritin was $100-200 \mu \mathrm{g} / \mathrm{L}$, it was administered only during the first dialysis session of the week. Patients with ferritin levels $201-700 \mu \mathrm{g} / \mathrm{L}$ and TSAT $\leq 20 \%$ received IV iron sucrose $100 \mathrm{mg}$ during the first dialysis session of the week. 
Follow-Up Assessments, Monitoring, and Ongoing Treatment

Patients were followed monthly for the duration of the trial. Laboratory tests assessed at each follow-up visit included hemoglobin, platelets, ferritin, TSAT, and albumin. The occurrence of adverse events, the need for blood transfusions, the status of vascular access, and the doses of ESA and iron were also recorded monthly. C-reactive protein concentrations were measured every 3 months, while QoL measures (i.e., EQ-5D QoL and KDQOL) were completed at months 3, 6, 9, 12, and every 6 months thereafter. Finally, red blood cell indices were collected at baseline and reassessed at 6-month intervals throughout the follow-up period.

IV iron was dosed as described above throughout the duration of the trial. If per-protocol doses of IV iron were not administered at the first HD session(s) in the week after monthly testing, they were administered at the next attended dialysis session. Investigators were instructed to withhold iron if the patient developed an active infection deemed sufficient to contraindicate the use of IV iron. In such cases, iron therapy was resumed when the investigator judged it safe.

Clinicians were instructed to adjust the dose of ESA therapy to maintain a target hemoglobin level of $10-12 \mathrm{~g} / \mathrm{dL}$. The maximum dose of ESA allowed was 30,000 international units of epoetin per week (or an average of $150 \mu \mathrm{g}$ per week of darbepoetin alfa or methoxypolyethylene glycol epoetin beta).

\section{Regulatory Considerations and Endpoint Adjudication}

The PIVOTAL trial is being conducted in compliance with the principles of the Declaration of Helsinki (1996), the principles of Good Clinical Practice, and in accordance with all applicable regulatory requirements. The study protocol was approved by the South East Coast - Brighton and Sussex Research Ethics Committee, and the Medicines and Healthcare products Regulatory Agency. All patients provided written informed consent.

The primary outcome and the first 6 secondary outcomes (i.e., components of the primary outcome) are identified from reports of death, hospital admissions, or other serious adverse events reported by the participant, study staff, treating physicians in primary or secondary care, or by computerized record linkage. All possible events will be documented, reviewed, and adjudicated by a blinded Endpoint Adjudication Committee (online suppl. Appendix 1; for all online suppl. material, see www.karger.com/ doi/10.1159/000493551) based on pre-specified criteria. An Independent Data Monitoring Committee will review safety data, including serious adverse events and the primary endpoint events, and will advise on acceptable continuation of the study, or whether the study should be stopped prematurely because of safety concerns.

\section{Powering and Sample Size}

Based on previous trials and registries (e.g., the AURORA trial [24] and the UK Renal Registry [25]), we expected the main component of the primary outcome would be death. In AURORA, the 1 -year mortality rate was approximately $14 \%$, and the annual rates of nonfatal MI and stroke were approximately 2 and 1\% respectively [24]. Similarly, the UK Renal Registry reported an $11.6 \%$ unadjusted 1-year mortality rate among incident HD patients (after 90 days) [25]. Therefore, initial sample size calculations assumed a 3-year primary event rate of $50 \%$ (the expected average follow-up in the present trial) in the control group.
Assuming a loss to follow-up rate of $10 \%$ (which includes renal transplantation), a total required sample size of 2,080 was calculated to allow rejection of a noninferiority hazard ratio (HR) limit of 1.20 with $80 \%$ power. After recruitment of 2,141 participants and blinded review of study endpoint rates, the steering committee decided to modify the noninferiority HR limit to 1.25 , requiring a minimum of 631 first primary endpoints to provide at least $80 \%$ power (online suppl. Appendix 2). Such a change aligns the PIVOTAL trial more closely with contemporary noninferiority clinical trials examining cardiovascular outcomes, including those reviewed by regulatory agencies in recent years [26, 27]. The change will also allow for completion of the trial in a reasonable time frame.

\section{Statistical Analysis}

Baseline characteristics will be summarized by randomized treatment group and for the overall cohort. The primary analyses will use the intention-to-treat population, defined as all validly randomized participants with follow-up censored at the end of study, date of withdrawal from study consent, date of loss-to-follow-up, or dates of renal transplantation, transfer to home dialysis or peritoneal dialysis, whichever comes first. Supporting analyses will be assessed in the per-protocol population, the population of randomized subjects that receive $\geq 1$ trial dose of iron and had no major protocol deviations in relation to the inclusion/exclusion criteria. As a pre-specified sensitivity analysis, noninferiority will also be assessed in the intention-to-treat population, with additional censoring of participants after discontinuation of study drug (i.e., an on-treatment analysis).

The primary endpoint (i.e., the composite of all-cause death, nonfatal MI, nonfatal stroke, or hospitalization for HF) will initially be analyzed to assess noninferiority between the comparator (proactive, high-dose iron) and control arm (reactive, low-dose iron). All analyses will be conducted using cause-specific Cox proportional hazards models including the stratification variables and the treatment variable. The HR for the treatment effect (proactive, high-dose arm compared to the reactive, low-dose arm) will be estimated with a $95 \%$ CI. A formal test (Wald statistic) of noninferiority will test the null hypothesis that the HR treatment effect is $\geq 1.25$ against the alternative that $\mathrm{HR}<1.25$, with a required significance level of 0.025 (one-sided test). If noninferiority is established, a two-sided superiority test (Wald statistic) will then be carried out. For the outcome involving recurrent events, the cumulative incidence of the composite events will be analyzed using the method of Ghosh and Lin [28] and the treatment groups will be compared using the proportional rate model of Lin et al. [29]. Additional analysis will be carried out on the composite nonfatal components of this outcome, using the same methodology. For the secondary outcomes involving time-to-first-event (i.e., components of the primary endpoint), superiority analyses will be carried out as described above, with no requirement for any $p$ value penalty. For all time-to-first-event analyses, cumulative incidence functions will be constructed for each outcome type adjusting for the competing risks of transplantation, transfer to home or peritoneal dialysis, and/or death as appropriate. The secondary analyses will be regarded as exploratory with no $p$ value adjustments for multiple comparisons.

Pre-specified sub-groups are time on dialysis (0-4 vs. 5-12 months), vascular access type (dialysis catheter vs. A-V fistulae), and diabetic status. Sub-group analyses will be carried out by add- 
ing the sub-grouping variable and its interaction with randomized treatment into the analysis models and testing for significance of the interaction terms.

Cumulative ESA dose requirements will be summarized by study visit and compared between treatment groups at each visit and overall (scaled by duration of follow-up) using stratified Wilcoxon rank sum tests. Time to first transfusion will be analyzed in a manner similar to the primary outcome. QoL scale and subscale scores will be compared via regression analyses adjusting for baseline levels. Similar analyses will be used to examine the effects of therapy on hemoglobin, iron indices, platelets, and albumin concentrations.

Safety outcomes of vascular access thrombosis and hospitalization will be analyzed in a manner similar to the primary efficacy outcome. The number of infection episodes will be analyzed using a negative binomial regression model adjusting for duration of follow-up. Adverse events will be summarized by Medical Dictionary for Regulatory Activities system organ class and preferred term and reported, but will not be subject to formal statistical testing.

\section{Results}

The PIVOTAL trial commenced in November 2013 and patients were recruited from 50 sites across the United Kingdom (online suppl. Appendix 1). A total of 2,589 patients were screened and 2,141 (83\%) were randomized before recruitment ended on October 4, 2016. By far, the most common reason for screening failure was a serum ferritin above $400 \mu \mathrm{g} / \mathrm{L}$, even after stopping IV iron. Among patients with high serum ferritin levels and/or TSAT levels at initial screening, 246 subsequently met eligibility criteria after a variable period of withholding iron (up to $~ 10$ months) and were then randomly assigned to a treatment arm. The required number of primary endpoints is projected to occur during mid-2018. At the time this manuscript was prepared, database lock has not occurred, and all data remain blinded.

\section{Baseline Characteristics of Randomized Participants}

Of the 2,141 randomized individuals, approximately two-thirds $(65.2 \%)$ were male (Table 3$)$. The median (lower and upper quartiles [LQ, UQ]) age was $65(52,75)$ years, with a range of 18-96 years. Seventy-nine percent of the randomized population was white. The median (LQ, UQ) weight was $80(67,95) \mathrm{kg}$ and most patients were classified as overweight or obese (median [LQ, UQ] body mass index of $28[24,33] \mathrm{kg} / \mathrm{m}^{2}$ ). As detailed in Table 3 , the causes of end-stage renal disease were typical of a dialysis population. At baseline, $56 \%$ had dialysis access through an arteriovenous fistula, $3 \%$ through a polytetrafluoroethylene graft, and $41 \%$ through a central venous catheter. Although the majority of patients had a hemo-
Table 3. Baseline characteristics of PIVOTAL randomized population

\begin{tabular}{|c|c|}
\hline Characteristic & $\begin{array}{l}\text { Randomized participants } \\
(n=2,141)\end{array}$ \\
\hline Age, years & $65(52,75)$ \\
\hline \multicolumn{2}{|l|}{ Gender, \% } \\
\hline Male & 65.3 \\
\hline Female & 34.7 \\
\hline \multicolumn{2}{|l|}{ Ethnicity, \% } \\
\hline White & 79 \\
\hline Black & 9 \\
\hline Asian & 9 \\
\hline Other & 3 \\
\hline Dialysis vintage, months & $4.8(2.8,8.2)$ \\
\hline$<5$ months, $\%$ & 46 \\
\hline$\geq 5$ months, $\%$ & 54 \\
\hline Diabetes, \% & 44 \\
\hline Hypertension, \% & 73 \\
\hline Prior MI, \% & 9 \\
\hline Atrial fibrillation, \% & 8 \\
\hline Prior stroke, \% & 8 \\
\hline Hyperlipidemia, \% & 25 \\
\hline \multicolumn{2}{|l|}{ Smoking status, $\%$} \\
\hline Current & 12 \\
\hline Former & 25 \\
\hline Never & 63 \\
\hline Weight, kg & $80(67,95)$ \\
\hline BMI, $\mathrm{kg} / \mathrm{m}^{2}$ & $28(24,33)$ \\
\hline Systolic BP, mm Hg ${ }^{\mathrm{a}}$ & $144(128,160)$ \\
\hline Diastolic BP, mm Hg & $73(64,83)$ \\
\hline Hemoglobin, g/dL & $10.6(9.6,11.5)$ \\
\hline Ferritin, $\mu \mathrm{g} / \mathrm{L}$ & $216(133,304)$ \\
\hline TSAT, $\%$ & $20(16,24)$ \\
\hline $\mathrm{CRP}, \mathrm{mg} / \mathrm{L}$ & $6(4,14)$ \\
\hline Albumin, g/L & $36(32,39)$ \\
\hline ESA dose, IU/week ${ }^{\mathrm{b}}$ & $8,000(5,000,12,000)$ \\
\hline \multicolumn{2}{|l|}{ Primary cause of renal failure, $\%$} \\
\hline Diabetic nephropathy & 33.4 \\
\hline Glomerular disease & 18.6 \\
\hline Hypertension & 11.0 \\
\hline Tubulointerstitial disease ${ }^{c}$ & 9.4 \\
\hline Renovascular disease & 6.9 \\
\hline Polycystic kidney disease & 5.5 \\
\hline Other & 6.1 \\
\hline Unknown & 9.2 \\
\hline
\end{tabular}

Continuous variables are shown as median (LQ, UQ).

${ }^{a}$ Blood pressure measurements represent pre-HD assessments.

b ESA dose for darbepoetin and methoxypolyethylene glycol epoetin beta converted to IU/week using standard conversion factors.

${ }^{\mathrm{c}}$ Includes pyelonephritis, reflux nephropathy, and obstructive uropathy.

BMI, body mass index; IU, international unit. 
globin between 10 and $12 \mathrm{~g} / \mathrm{dL}$ at baseline, $>25 \%$ of the cohort had a hemoglobin concentration $<10 \mathrm{~g} / \mathrm{dL}$. Consistent with the inclusion criteria (i.e., ferritin $<400 \mathrm{ug} / \mathrm{L}$ ), the median (LQ, UQ) ferritin level was $216(133,304)$ $\mu \mathrm{g} / \mathrm{L}$. According to inclusion criteria, all patients were receiving ESA, with 54\% receiving darbepoetin alfa. The remaining patients were receiving epoetin alfa (25\%), epoetin beta (17\%), methoxy polyethylene glycol-epoetin beta $(4 \%)$, and epoetin theta $(<0.01 \%)$.

\section{Prior Cardiovascular Events and Risk Factors}

A history of major adverse cardiovascular events was rare among participants, reflecting the incident nature of the population; prior stroke and MI were present in 8 and $9 \%$ of the cohort respectively. A history of HF or peripheral vascular disease was recorded for 4 and $9 \%$ of participants respectively. Approximately $8 \%$ of the randomized population had a history of atrial fibrillation and one-quarter were diagnosed with hyperlipidemia. Nearly two-thirds of participants (63\%) denied any history of cigarette smoking. As would be expected for a dialysis population, $73 \%$ of the cohort had hypertension. At baseline, $44 \%$ of patients had diabetes.

\section{Discussion}

The 2 components of anemia management in HD patients are ESA therapy and IV iron. In contrast to ESA therapy, where several high-profile randomized controlled trials inform clinical practice and clinical guidelines $[8,30-32]$, there are no trials that are sufficiently robust to inform physicians on the use of IV iron in HD patients, despite this being standard care. Because of the lack of evidence, there is a large disparity in the quantities of IV iron administered to patients maintained on chronic HD [3]. There are both potential benefits and harms in using large amounts of IV iron in this patient population, such that there is clinical equipoise, which is an ideal, if not essential, prerequisite for a randomized controlled trial.

PIVOTAL was designed to investigate the noninferiority of a proactive, high-dose IV iron regimen compared with a reactive, low-dose regimen and has $80 \%$ power to exclude a $25 \%$ increase in the HR for hard clinical endpoints including death and nonfatal cardiovascular events. In addition to the individual events making up the primary endpoint, there are also a number of important pre-specified secondary endpoints, including hospitalizations, infections (infectious-related hospitalizations, deaths due to infections), ESA dose and transfusion requirements, and QoL measures. We have completed recruitment and are on course to reach the required number of endpoints in mid-2018. After appropriate clinical data management (e.g., data validation), the database will be locked, and statistical analysis will begin.

In selecting the iron doses for the proactive, high-dose and reactive, low-dose arms, there had to be a clear separation between the treatment regimens. However, there are also ethical considerations and the need to be sensitive to what is presently regarded as acceptable practice. For the reactive, low-dose arm, the trial design was sensitive to the national and international guidelines on anemia management with respect to the lowest acceptable iron limits, namely, a ferritin of $200 \mu \mathrm{g} / \mathrm{L}$ and TSAT of $20 \%$. Thus, it was intended that patients randomized to this arm should have their iron status maintained just above these thresholds, but not greatly exceeding them. For the proactive, high-dose arm, a safety cut-off beyond which no more IV iron should be given, was needed. In order to ensure investigator comfort and "buy-in," and therefore success in this multicenter trial, a survey was conducted in 2012 at a national meeting of renal healthcare professionals in the United Kingdom. Attendees were asked about ferritin levels above which they would be concerned about administering a significant amount of additional IV iron on a monthly basis. The majority decision was a ferritin cutoff of $700 \mu \mathrm{g} / \mathrm{L}$, and this was the selected threshold for the PIVOTAL study protocol. Above this level it was proposed that IV iron would be temporarily withheld for that month. There are inevitable limitations to selecting these thresholds, but the study is sufficiently powered, scientifically rigorous, and likely to inform preferred clinical practice for IV iron administration in the future.

The baseline demographic data of the PIVOTAL trial cohort align very closely with the "real world" dialysis population in the United Kingdom as detailed by the latest data from the UK Renal Registry (online suppl. Table 1). Baseline age, sex, body mass index, blood pressure, diabetes prevalence, and the proportion of current smokers are all similar (R Steenkamp, unpublished data, June 2018). There are slightly more white patients in the trial population (79 vs. $72 \%$ ), possibly reflecting the known under-representation of ethnic minorities in clinical trials [33-36]. Baseline laboratory variables are also representative of the broader dialysis population in the United Kingdom, with the exception of the serum ferritin level which, as mandated by the PIVOTAL protocol, had to be $<400 \mu \mathrm{g} / \mathrm{L}$ to qualify for enrollment. It is reassuring to see 
that the trial population is indeed representative of the "real world" dialysis population in the United Kingdom, and fairly similar to most dialysis populations worldwide [37-42]. There are, of course, a far higher proportion of white patients in the study, compared with dialysis populations in the United States and Asia, and some differences in the rates of comorbid conditions (e.g., diabetes), which are other minor limitations of the trial.

Despite these limitations, the study should be able to answer several important research questions. The primary analysis will be a noninferiority analysis to ascertain whether the proactive, high-dose arm has any safety signals over the reactive, lower dose arm. If noninferiority is satisfied, then the next statistical analysis will be a superiority analysis. Clearly, not all possible outcomes need be positive or negative. Thus, results for a comparison of the primary endpoint may be different from results of an important secondary endpoint, such as infections. Nevertheless, the study has considerable power to examine these important primary and secondary endpoints.

In conclusion, PIVOTAL is the first rigorously designed scientific study to examine the issue of IV iron dosing in HD patients. It is likely that the results of this trial, which are expected to be available towards the end of 2018, will yield information relevant to every dialysis physician and dialysis provider worldwide, for the benefit of patients.

\section{Acknowledgments}

Medical writing support was provided by Adam Perahia, MD, of NorthStar Strategic Consulting LLC via funding by Kidney Research UK, which is supported by an unrestricted grant from Vifor Fresenius Medical Care Renal Pharma Ltd.

The authors thank the nurses and staff at each of the dialysis centers participating in the trial. We are also grateful to all the patients who participated in this study.

\section{Disclosure Statements}

I.C.M. has received speaker fees, honoraria, and consultancy fees from several ESA and IV iron manufacturers, including Akebia, AMAG, Astellas, Bayer, FibroGen, GlaxoSmithKline, Pharmacosmos, and Vifor Pharma. C.W., K.F., J.J.V.M., H.M., R.S., and C.R.V.T. have no conflicts of interest. S.D.A. has received grants from Vifor Pharma and Abbott Vascular, and fees for consultancy from Vifor Pharma, Bayer, Boehringer Ingelheim, Novartis, and Servier. S.B. has received speaker fees, honoraria, and consultancy fees from Pharmacosmos and Vifor Pharma. P.A.K. has received speaker fees, honoraria, and consultancy fees from Pharmacosmos, Vifor Pharma, and Takeda. D.C.W. has received honoraria and consultancy fees from Amgen, Akebia, AstraZeneca, Bayer, Boehringer Ingelheim, Janssen, Napp, and Vifor Fresenius Medical Care. C.G.W. has received a research grant from Roche. I.F. has received research grants from Vifor Pharma and Pharmacosmos. The results presented in this paper have not been published previously in whole or in part, except in abstract form.

\section{Funding Sources}

The PIVOTAL trial is funded by Kidney Research UK, which is supported by an unrestricted grant from Vifor Fresenius Medical Care Renal Pharma Ltd. However, this is an academic-led trial, and the company had no input into the study design or delivery of the trial. They were, however, kept abreast of the progress of the trial via regular study reports and newsletters. Vifor Fresenius Medical Care Renal Pharma Ltd. also provided iron sucrose for the trial, free of charge.

\section{Authors' Contributions}

I.C.M. conceived the study, contributed to the study design and statistical analysis plan, and developed the first draft of the manuscript, which was critically reviewed and revised by the other authors. All other authors contributed to the development of the study design and statistical analysis plan. I.F. provided biostatistical support.

\section{References}

1 Macdougall IC, Bircher AJ, Eckardt KU, et al: Iron management in chronic kidney disease: conclusions from a "Kidney Disease: Improving Global Outcomes" (KDIGO) Controversies Conference. Kidney Int 2016;89:28-39.

2 Wish JB, Aronoff GR, Bacon BR, et al: Positive iron balance in chronic kidney disease: how much is too much and how to tell? Am J Nephrol 2018;47:72-83.

3 Bailie GR, Larkina M, Goodkin DA, et al: Variation in intravenous iron use internation- ally and over time: the Dialysis Outcomes and Practice Patterns Study (DOPPS). Nephrol Dial Transplant 2013;28:2570-2579.

4 Locatelli F: Iron treatment and the TREAT trial. NDT Plus 2011;4(suppl 1):i3-i5.

5 Szczech LA, Barnhart HX, Inrig JK, et al: Secondary analysis of the CHOIR trial epoetinalpha dose and achieved hemoglobin outcomes. Kidney Int 2008;74:791-798.

6 McCullough PA, Barnhart HX, Inrig JK, et al: Cardiovascular toxicity of epoetin-alfa in pa- tients with chronic kidney disease. Am J Nephrol 2013;37:549-558.

7 Kilpatrick RD, Critchlow CW, Fishbane S, et al: Greater epoetin alfa responsiveness is associated with improved survival in hemodialysis patients. Clin J Am Soc Nephrol 2008;3: 1077-1083.

8 Pfeffer MA, Burdmann EA, Chen CY, et al: A trial of darbepoetin alfa in type 2 diabetes and chronic kidney disease. N Engl J Med 2009; 361:2019-2032. 
9 Skali H, Parving HH, Parfrey PS, et al: Stroke in patients with type 2 diabetes mellitus, chronic kidney disease, and anemia treated with Darbepoetin Alfa: the trial to reduce cardiovascular events with Aranesp therapy (TREAT) experience. Circulation 2011;124: 2903-2908.

10 Goodkin DA: The normal hematocrit cardiac trial revisited. Semin Dial 2009;22:495-502.

11 Solomon SD, Uno H, Lewis EF, et al: Erythropoietic response and outcomes in kidney disease and type 2 diabetes. N Engl J Med 2010; 363:1146-1155.

12 Ponikowski P, van Veldhuisen DJ, CominColet J, et al: Beneficial effects of long-term intravenous iron therapy with ferric carboxymaltose in patients with symptomatic heart failure and iron deficiency. Eur Heart J 2015; 36:657-668.

13 Anker SD, Comin Colet J, Filippatos G, et al: Ferric carboxymaltose in patients with heart failure and iron deficiency. N Engl J Med 2009;361:2436-2448.

14 Toblli JE, Di Gennaro F, Rivas C: Changes in echocardiographic parameters in iron deficiency patients with heart failure and chronic kidney disease treated with intravenous iron. Heart Lung Circ 2015;24:686-695.

15 Jankowska EA, Tkaczyszyn M, Suchocki T, et al: Effects of intravenous iron therapy in irondeficient patients with systolic heart failure: a meta-analysis of randomized controlled trials. Eur J Heart Fail 2016;18:786-795.

16 Anker SD, Kirwan BA, van Veldhuisen DJ, et al: Effects of ferric carboxymaltose on hospitalisations and mortality rates in iron-deficient heart failure patients: an individual patient data meta-analysis. Eur J Heart Fail 2018;20:125-133.

17 Ganguli A, Kohli HS, Khullar M, Lal Gupta K Jha V, Sakhuja V: Lipid peroxidation products formation with various intravenous iron preparations in chronic kidney disease. Ren Fail 2009;31:106-110.

18 Del Vecchio L, Longhi S, Locatelli F: Safety concerns about intravenous iron therapy in patients with chronic kidney disease. Clin Kidney J 2016;9:260-267.

19 Malindretos P, Sarafidis PA, Rudenco I, et al: Slow intravenous iron administration does not aggravate oxidative stress and inflammatory biomarkers during hemodialysis: a comparative study between iron sucrose and iron dextran. Am J Nephrol 2007;27:572579 .

20 Drakesmith H, Prentice AM: Hepcidin and the iron-infection axis. Science 2012;338: 768-772.

21 Hougen I, Collister D, Bourrier M, et al: Safety of intravenous iron in dialysis: a systematic review and meta-analysis. Clin J Am Soc Nephrol 2018;13:457-467.

22 Ishida JH, Johansen KL: Iron and infection in hemodialysis patients. Semin Dial 2014;27: 26-36.

23 Li X, Kshirsagar AV: Rest easy with intravenous iron for dialysis patients? Clin J Am Soc Nephrol 2018;13:363-365.

24 Fellstrom BC, Jardine AG, Schmieder RE, et al: Rosuvastatin and cardiovascular events in patients undergoing hemodialysis. N Engl J Med 2009;360:1395-1407.

25 Methven S, Steenkamp R, Fraser S: UK Renal Registry 19th Annual Report: Chapter 5 Survival and Causes of Death in UK Adult Patients on Renal Replacement Therapy in 2015: National and Centre-specific Analyses. Nephron 2017;137(suppl 1):117-150.

26 Center for Drug Evaluation and Research. Guidance for Industry - Diabetes mellitus: Evaluating Cardiovascular Risk in New Antidiabetic Therapies to Treat Type 2 Diabetes. December 2008. https://www.fda.gov/ downloads/Drugs/.../Guidances/ ucm071627.pdf

27 Marchenko O, Jiang Q, Chuang-Stein C, et al: Statistical considerations for cardiovascular outcome trials in patients with type 2 diabetes mellitus. Stat Biopharm Res 2017;9:347-360.

28 Ghosh D, Lin DY: Nonparametric analysis of recurrent events and death. Biometrics 2000; 56:554-562.

29 Lin DY, Wei LJ, Yang I, Ying Z: Semiparametric regression for the mean and rate functions of recurrent events. J R Stat Soc B 2000;62: 711-730.

30 Singh AK, Szczech L, Tang KL, et al: Correction of anemia with epoetin alfa in chronic kidney disease. N Engl J Med 2006;355:20852098.

31 Drueke TB, Locatelli F, Clyne N, et al: Normalization of hemoglobin level in patients with chronic kidney disease and anemia. N Engl J Med 2006;355:2071-2084.
32 Besarab A, Bolton WK, Browne JK, et al: The effects of normal as compared with low hematocrit values in patients with cardiac disease who are receiving hemodialysis and epoetin. N Engl J Med 1998;339:584-590.

33 Geller SE, Koch AR, Roesch P, Filut A, Hallgren $\mathrm{E}$, Carnes $\mathrm{M}$ : The more things change, the more they stay the same: a study to evaluate compliance with inclusion and assessment of women and minorities in randomized controlled trials. Acad Med 2018;93:630-638.

34 George S, Duran N, Norris K: A systematic review of barriers and facilitators to minority research participation among African Americans, Latinos, Asian Americans, and Pacific Islanders. Am J Public Health 2014;104:e16e31.

35 Hussain-Gambles M, Atkin K, Leese B: Why ethnic minority groups are under-represented in clinical trials: a review of the literature. Health Soc Care Community 2004;12:382388

36 Rathore SS, Krumholz HM: Race, ethnic group, and clinical research. BMJ 2003;327: 763-764.

37 United States Renal Data System: 2017 USRDS Annual Data Report: Epidemiology of Kidney Disease in the United States. National Institutes of Health, National Institute of Diabetes and Digestive and Kidney Diseases, Bethesda, MD, 2017.

38 Byrne C, Caskey F, Castledine C, et al: UK renal registry 19th annual report. Nephron 2017;137(suppl 1):1-406.

39 Young EW, Goodkin DA, Mapes DL, et al: The Dialysis Outcomes and Practice Patterns Study (DOPPS): an international hemodialysis study. Kidney Int 2000;57:S74-S81.

40 Duranton F, Kramer A, Szwarc I, et al: Geographical variations in blood pressure level and seasonality in hemodialysis patients. Hypertension 2018;71:289-296.

41 Goodkin DA, Bragg-Gresham JL, Koenig KG, et al: Association of comorbid conditions and mortality in hemodialysis patients in Europe, Japan, and the United States: the Dialysis Outcomes and Practice Patterns Study (DOPPS). J Am Soc Nephrol 2003;14:3270-3277.

42 US Dialysis Outcomes and Practice Patterns Study (DOPPS) Practice Monitor, April 2018. http://www.dopps.org/DPM (accessed July 7, 2018). 